\title{
Prática, Desafios e Expectativas da Supervisão de Estágio em Medicina
}

\author{
Practice, Challenges and Expectations of Probation Supervision in Medicine
}

Práctica, Desafíos y Expectativas de Supervisión de Libertad Condicional en Medicina

Rosana Quintella Brandão Vilela ${ }^{1}$

Daniel Pinheiro Jucá ${ }^{2}$

\section{Resumo}

Objetivo: investigar a prática do supervisor e preceptor durante o acompanhamento dos estagiários, no internato hospitalar de medicina. Método: Trata-se de um estudo descritivo do tipo transversal, baseado na aplicação de um $\begin{array}{llll}\text { questionário junto a } & 14\end{array}$ preceptores/supervisores do estágio hospitalar obrigatório do Curso de Medicina de uma Universidade Pública de Alagoas. Resultados: preceptores/supervisores tem mais de 20 anos de graduação, poucos têm formação específica para a docência em saúde e dedicam de 13 a 24 horas semanais à esta função. A maioria realiza atividades que envolvem, predominantemente, os trabalhos isolados entre preceptor/supervisor e aluno, e entre o preceptor/supervisor, aluno e paciente/usuário fora do contexto interdisciplinar e interprofissional. Conclusões: Torna-se necessário investimento em uma prática pedagógica mais problematizadora e significativa capaz de provocar nestes docentes e, consequentemente, nos estudantes, novas reflexões, novos caminhos, novos sentidos para uma educação interprofissional e o exercício da EPS.

\section{Palavras-chaves: Medicina; Educação Superior; Estágio Clínico.}

\section{Abstract}

Objective: To investigate the supervisor and tutor practice during the 
monitoring of trainees during the medical intern medicine. Method: This is a descriptive transversal study, based on the application of a questionnaire to 14 preceptors/supervisors from hospital training of the Medical School of the Public University of Alagoas. Results: preceptors/supervisors with more than 20 years of graduation, few have specific training for teachers in health and devote 13-24 hours per week to this function. Most conducts activities involving, predominantly, the work isolated between preceptor/supervisor and student, and between the preceptor/supervisor, student and patient/user outside the context interdisciplinary and interprofissional. Conclusion: The study's findings show that it is needed more investment in a more problematical and significant pedagogical practice capable of provoking these teachers and, consequently, the students, new thinking, new ways, new directions for interprofissional education and exercise of EPS.

Keywords: Medicine; Higher Education; Clinical Clerkship.

\section{Resumen}

Objetivo: investigar el supervisor de la práctica y preceptor durante el seguimiento de los participantes durante la medicina médico interno. Método: Se trata de un estudio descriptivo transversal, basado en la aplicación de un cuestionario a 14 preceptores/supervisores requerido curso de formación del hospital de Medicina de una Universidad Pública de Alagoas. Resultados: los preceptores/supervisores tienen más de 20 años de graduación, pocos tengan una formación específica para los profesores en materia de salud y dedica 13-24 horas por semana a esta función. La mayoría realiza actividades que implica, principalmente, los trabajos aislados entre preceptor/supervisor $y$ estudiante, $y$ entre el preceptor/supervisor, estudiante $y$ paciente/usuario fuera de contexto interdisciplinario e interprofesional. Conclusión: El estudio mostró que es necesario invertir en la enseñanza hacia una práctica pedagógica más problemática y significativa capaz de causar en estos maestros y, en consecuencia, en los estudiantes, nиevos pensamientos, nuеvos caminos, nuevas orientaciones para una educación interprofesional y el ejercicio de EPS. 
Palabras clave: Medicina; Educación Superior; Prácticas Clínicas.

\section{Introdução}

A formação acadêmica de profissionais da área de saúde no Brasil deve ser orientada aos princípios e diretrizes do Sistema Único de Saúde (SUS), para que, de fato, as estratégias de cuidado da saúde sejam direcionadas às reais demandas de saúde da população. Para tanto, as Instituições de Ensino Superior (IES) devem direcionar os currículos de seus cursos às necessidades do Sistema ${ }^{(1-4)}$.

A qualificação dos recursos humanos na área de saúde tem demandado esforços conjuntos dos Ministérios da Saúde e da Educação, que culminaram com a elaboração das novas Diretrizes Curriculares Nacionais dos cursos de graduação na área da saúde (DCN saúde), que recomendam a formação voltada ao SUS, o perfil generalista do egresso, bem como as competências necessárias a estes profissionais, respeitando-se as peculiaridades de cada profissão ${ }^{(5-7)}$.

Nesse sentido, o estágio supervisionado (internato) que ocorre

durante os últimos anos do curso de medicina, é o espaço privilegiado da prática, durante a graduação e caracteriza-se pelo desenvolvimento de competências, conhecimentos teóricoconceituais, habilidades e atitudes - em situações de aprendizagem, conduzidas no ambiente profissional ${ }^{(8)}$.

A legislação nacional estabelece que tais estágios sejam realizados em cenários diversificados, nos níveis hierárquicos de atenção à saúde do Sistema Único de Saúde. E, durante os mesmos, o formando tem contato direto com a prática de sua profissão, aprende a aprender de forma interprofissional com a rede de saúde e com a comunidade $^{(7,9,10)}$.

Percebe-se que o estudo criterioso e sistemático do estágio obrigatório em Medicina é um dos principais caminhos para se compreender melhor como se dá a formação desse profissional. Diante disso, e sabendo-se que as DCN de medicina reafirmam a necessidade de se trabalhar durante a graduação com a $\operatorname{EPS}^{(7,9,10)}$, esta investigação teve como objetivos investigar a prática do supervisor e preceptor durante $\mathrm{o}$ acompanhamento dos estagiários.

\section{Método}


Por se tratar de um estudo que envolve seres humanos, o estudo foi realizado após aprovação pelo Comitê de Ética em Pesquisa e Ensino do Centro Universitário CESMAC (protocolo $\mathrm{n}^{\mathrm{o}} 1309 / 12$ ). Todos os sujeitos que se disponibilizaram a colaborar receberam esclarecimentos sobre a pesquisa, assinaram o Termo de Consentimento Livre e Esclarecido (TCLE) e responderam à entrevista de forma individual e sigilosa.

Foi realizado um estudo quantitativo transversal descritivo, baseado na aplicação de um questionário estruturado junto aos preceptores e/ou supervisores do Internato do curso de Medicina de uma IES pública, federal do estado de Alagoas, durante o ano de 2013. Para essa pesquisa, o termo preceptor representa o Médico (técnico) e/ou docente que acompanha os internos (estagiários do $9^{\circ}$ ao $12^{\circ}$ período) nos cenários de prática, e o termo supervisor de estágio representa o Médico (técnico) e/ou docente que é responsável pela coordenação das atividades dos demais preceptores relacionados a um determinado estágio $^{(11)}$. O preceptor é o responsável por acompanhar, orientar, observar e avaliar os alunos, bem como registrar suas frequências, discutir os casos e elaborar os relatórios durante o estágio, enquanto que o supervisor é o responsável por determinada área específica de estágio, que planeja, organiza e orienta o aluno em intercâmbio com o os demais preceptores de um mesmo estágio ${ }^{(12)}$.

A amostra do presente estudo foi composta por 14 preceptores e/ou supervisores que atuam nas diversas áreas do internato de Medicina, em ambiente hospitalar. Foram entrevistados 14 médicos pertencentes a quatro das cinco grandes áreas da Medicina, sendo: 03 da clínica médica, 02 da cirurgia, 05 da pediatria e 04 da ginecologia e obstetrícia.

A Tabela 1 traz a caracterização da amostra com relação ao gênero, faixa etária, tipo de vínculo, cargo e função exercidos e áreas de atuação.

O Estágio Supervisionado Obrigatório (internato) em Medicina tem parte de suas atividades realizada em ambiente hospitalar, e este foi o campo desta pesquisa, tendo como sujeitos, preceptores e/ou supervisores de grandes áreas da medicina como: Clínica Médica, Cirurgia, GinecologiaObstetrícia e Pediatria. 
Tabela 1: Caracterização da amostra com relação ao gênero, faixa etária, tipo de vínculo, cargo exercido, função exercida e área de atuação.

\begin{tabular}{lcc}
\hline Variável & $\mathbf{N}=\mathbf{1 4}$ & $\mathbf{\%}$ \\
\hline Gênero & & \\
Feminino & 09 & 64,28 \\
Masculino & 05 & 35,72 \\
& & \\
Faixa etária & & \\
31 a 40 & 03 & 21,42 \\
41 a 50 & 05 & 35,71 \\
>50 & 06 & 42,85 \\
& & \\
Tipo de Vínculo & & \\
Efetivo/concursado & 14 & 100 \\
& & \\
Cargo Exercido & & \\
Docente & 11 & 78,57 \\
Técnico (Médico) & 01 & 7,14 \\
Técnico e Docente & 02 & 14,28 \\
& & \\
Função exercida & & \\
Preceptoria apenas & 11 & 78,57 \\
Supervisão apenas & 00 & 0 \\
Preceptoria e Supervisão & 03 & 21,42 \\
& & \\
Grande Área de Atuação & & \\
Clínica Médica & 03 & 21,42 \\
Cirurgia & \\
Ginecologia e Obstetrícia \\
Pediatria & 02 & 14,28 \\
\hline Fonte: Elaborado pelo autor, 2013. & \\
& 05 & 35,71 \\
\hline
\end{tabular}

A pesquisa teve como instrumento utilizado o questionário estruturado - adaptado dos trabalhos de Missaka $^{(9)}$ e Trajman ${ }^{(13)}$, aplicado pelo pesquisador junto aos preceptores e/ou supervisores que concordaram em participar da pesquisa. O mesmo contemplou questões objetivas relacionadas à caracterização do profissional, como função exercida, cargo exercido e tipo de vínculo que possui com a instituição; ao percurso acadêmico, como tempo de formação, titulação e preparo didático específico para a docência; e por fim, relacionadas ao exercício da atividade de preceptoria e/ou supervisão propriamente ditos, contemplando tempo de exercício, carga horária semanal, razões de ingresso na função, tipo de atividade desenvolvida, como se dá o planejamento, quais as 
ações cotidianas e o grau de satisfação

com a mesma.

\section{Resultados}

Após a coleta, os dados foram armazenados em planilha eletrônica Sobre a formação docente dos (Microsoft Excel 2007®. Redmond, preceptores e/ou supervisores a tabela 2 WA, EUA) na forma de banco de apresenta os dados obtidos sobre o dados. Os resultados foram tabulados e percurso acadêmico dos preceptores as frequências das variáveis de cada e/ou supervisores estudados. grupo foram calculadas e dispostas nas formas gráfica e tabula.

Tabela 2: Percurso acadêmico dos preceptores e/ou supervisores.

\begin{tabular}{|c|c|c|}
\hline Variável & $\mathbf{N}$ & $\%$ \\
\hline Tempo decorrido após a graduação & $(\mathrm{N}=14)$ & \\
\hline 6 a 10 anos & 02 & 14,28 \\
\hline 11 a 20 anos & 04 & 28,57 \\
\hline 21 a 30 anos & 05 & 35,71 \\
\hline Maior que 30 anos & 03 & 21,42 \\
\hline Pós-graduação & $(\mathrm{N}=14)$ & \\
\hline Sim & 14 & 100 \\
\hline Não & 00 & 0 \\
\hline Maior Titulação & $(\mathrm{N}=14)$ & \\
\hline Residência & 03 & 21,42 \\
\hline Mestrado & 04 & 28,57 \\
\hline Doutorado & 07 & 50 \\
\hline Preparo didático para a docência & $(\mathrm{N}=14)$ & \\
\hline Sim & 11 & 78,57 \\
\hline Não & 03 & 21,42 \\
\hline Tipo de preparo* & $(\mathrm{N}=\mathbf{1 1})$ & \\
\hline 1- Aperfeiçoamento específico para docência. & 01 & 9,09 \\
\hline 2- Disciplina de didática durante o mestrado. & 05 & 45,45 \\
\hline $\begin{array}{l}\text { 3- Aperfeiçoamento específico para docência e especialização específica para } \\
\text { docência. }\end{array}$ & 02 & 18,18 \\
\hline $\begin{array}{l}\text { 4- Aperfeiçoamento específico para docência e disciplina de didática durante o } \\
\text { mestrado. }\end{array}$ & 01 & 9,09 \\
\hline $\begin{array}{l}\text { 5- Especialização específica para docência e disciplina de didática durante o } \\
\text { mestrado. }\end{array}$ & 01 & 9,09 \\
\hline $\begin{array}{l}\text { 6- Disciplina de didática durante o mestrado e disciplina de didática durante o } \\
\text { doutorado. }\end{array}$ & 01 & 9,09 \\
\hline Escolheu mais de uma resposta & 05 & 45,45 \\
\hline
\end{tabular}

Fonte: Elaborado pelo autor, 2013. 
No que concerne ao percurso acadêmico, o tempo decorrido após a graduação do grupo estudado foi superior a 21 anos $(57,14 \%)$.

Todos os entrevistados afirmaram possuir pós-graduação, sendo o doutorado referido por $50 \%$ destes.

O preparo didático específico para a docência, foi referido por onze preceptores/supervisores $(78,57 \%)$, que afirmaram tê-lo realizado por meio de disciplinas ministradas em cursos de aperfeiçoamento, de especialização ou durante o mestrado ou doutorado. Cerca de $45,45 \%$ responderam que se submeteram a mais de um tipo de preparo didático para a docência.

Sobre a rotina da função dos docentes, a tabela 3 apresenta o cenário encontrado entre os preceptores e/ou supervisores nos aspectos de razão do ingresso na atividade de preceptoria e/ou supervisão, tempo de exercício da função, carga horária semanal e número médio de internos preceptorados por vez.

Tabela 3: Razão do ingresso na atividade de preceptoria e/ou supervisão, tempo de exercício da função, carga horária semanal e número médio de internos preceptorados por vez.

\begin{tabular}{|c|c|c|}
\hline Variável & $\mathbf{N}=14$ & $\%$ \\
\hline \multicolumn{3}{|l|}{ Razão do ingresso na preceptoria } \\
\hline Escolha própria & 08 & 57,14 \\
\hline Determinação da chefia & 03 & 21,42 \\
\hline Atividade prevista nas atribuições de docência & 01 & 7,14 \\
\hline É um cargo inerente a quem presta serviço na especialidade abordada & 01 & 7,14 \\
\hline Não se recorda & 01 & 7,14 \\
\hline \multicolumn{3}{|l|}{ Tempo de exercício da função } \\
\hline 1 a 9 anos & 07 & 50 \\
\hline 10 a 19 anos & 03 & 21,42 \\
\hline 20 a 29 anos & 04 & 28,57 \\
\hline \multicolumn{3}{|l|}{ Carga horária semanal } \\
\hline Menor ou igual a 5 & 02 & 14,28 \\
\hline 6 a 12 & 05 & 35,71 \\
\hline 13 a 24 & 07 & 50 \\
\hline 25 a 30 & 00 & 0 \\
\hline$>30$ & 00 & 0 \\
\hline \multicolumn{3}{|l|}{ Número médio de internos preceptorados por vez } \\
\hline 01 a 05 internos & 04 & 28,57 \\
\hline 06 a 10 internos & 08 & 57,14 \\
\hline Maior que 10 internos & 02 & 14,28 \\
\hline Média geral igual a 7 & & \\
\hline
\end{tabular}


Sobre a atividade de preceptoria propriamente dita, quando os docentes foram questionados com relação ao ingresso na função, a principal razão apontada pelos participantes foi a escolha própria $(57,14 \%)$ seguida de determinação da chefia.

Em relação ao tempo na função de preceptor e/ou supervisor, $50 \%$ dos pesquisados encontram-se na fase inicial (de 1 a 9 anos).

Metade dos preceptores e/ou supervisores entrevistados dedica de 13 a 24 horas semanais à tal atividade e apenas $14,28 \%$ dedica carga semanal inferior ou igual a 5 horas, sendo responsável em média por 07 internos por turno de preceptoria - variando de 01 (pediatria) a 14 internos (clínica médica).

Com relação ao grau de satisfação diante da função exercida, a maioria dos profissionais $(64,28 \%)$ se consideram satisfeitos ou muito satisfeitos com as atividades de preceptoria que desenvolvem.

A caracterização do trabalho de preceptoria/supervisão do grupo de docentes pesquisados e da atividade de supervisão quanto ao tipo de atividade desenvolvida, modo de planejamento da atividade e ações realizadas durante a preceptoria e/ou supervisão, encontra-se na tabela 4.

Neste momento da pesquisa, os preceptores e/ou supervisores poderiam escolher as alternativas que representassem as atividades/ações realizadas durante preceptoria/supervisão e sua prática cotidiana e o modo como a planeja, portanto, muitos deles responderam mais de uma opção como resposta a estes questionamentos.

Os tipos de atividades mais desenvolvidos, segundo os profissionais pesquisados, foram trabalho isolado I (envolvendo apenas preceptor/supervisor e aluno) e, quase que a totalidade da amostra, o trabalho isolado II (composto por preceptor/supervisor, aluno e paciente/usuário).

Apenas uma vez referida a participação dos gestores nas reuniões. Já o modo de planejamento das atividades predominante foi o Trabalho isolado com a participação de preceptor e/ou supervisor e o aluno, sendo referido de forma unânime pelos entrevistados. 
Tabela 4: Caracterização do trabalho de preceptoria/supervisão do grupo de docentes pesquisados e da atividade de supervisão quanto ao tipo de atividade desenvolvida, modo de planejamento da atividade e ações realizadas durante a preceptoria e/ou supervisão.

\begin{tabular}{|c|c|c|}
\hline Variável & $\mathrm{N}=14$ & $\%$ \\
\hline \multicolumn{3}{|l|}{ Atividades/ações realizadas durante a preceptoria* } \\
\hline Indicação de leitura complementar & 14 & 100 \\
\hline Discussão de artigo e/ou capítulo de livro & 08 & 57,14 \\
\hline Revisão/Estudo de prontuário & 10 & 71,42 \\
\hline Demonstração de técnicas e procedimentos & 14 & 100 \\
\hline Explicação oral do preceptor e/ou supervisor & 14 & 100 \\
\hline Discussão de caso clínico & 03 & 21,42 \\
\hline Seminários & 06 & 42,85 \\
\hline Escolheu mais de uma opção & 14 & 100 \\
\hline \multicolumn{3}{|l|}{ Tipo de atividade desenvolvida na rotina } \\
\hline Trabalho isolado I [preceptor/supervisor + aluno] & 09 & 64,28 \\
\hline Trabalho isolado II [preceptor/supervisor + aluno + paciente/usuário] & 13 & 92,85 \\
\hline Trabalho em equipe I [preceptor/supervisor + residente + aluno] & 01 & 7,14 \\
\hline $\begin{array}{l}\text { Trabalho em equipe II [preceptor/supervisor + médico assistente + aluno + } \\
\text { paciente/usuário] }\end{array}$ & 02 & 14,28 \\
\hline $\begin{array}{l}\text { Trabalho em equipe III [preceptor/supervisor + profissional médico de } \\
\text { especialidade diferente }+ \text { aluno] }\end{array}$ & 01 & 7,14 \\
\hline Trabalho em equipe IV [preceptor/supervisor + profissionais de outras áreas que & & \\
\hline não médica + aluno] & 01 & 7,14 \\
\hline $\begin{array}{l}\text { Trabalho em equipe V [preceptor/supervisor + profissionais de outras áreas que } \\
\text { não médica + aluno + paciente/usuário] }\end{array}$ & 03 & 21,42 \\
\hline $\begin{array}{l}\text { Trabalho em equipe VI [preceptor/supervisor + profissionais de outras áreas que } \\
\text { não médica + residente + aluno + paciente + usuário] }\end{array}$ & 01 & 7,14 \\
\hline $\begin{array}{l}\text { Trabalho em equipe VII [preceptor/supervisor + alunos + gestores + } \\
\text { paciente/usuário] }\end{array}$ & 01 & 7,14 \\
\hline \multicolumn{3}{|l|}{ Modo de planejamento da atividade desenvolvida } \\
\hline Construção própria [preceptor/supervisor] & 07 & 50 \\
\hline Trabalho isolado [preceptor/supervisor + aluno] & 14 & 100 \\
\hline $\begin{array}{l}\text { Trabalho em equipe [preceptor/supervisor + profissionais de outras áreas que não } \\
\text { médica + aluno] }\end{array}$ & 01 & 7,14 \\
\hline
\end{tabular}

Outra forma importante de planejamento foi a construção própria das atividades, escolhidas por metade da amostra.

\section{Discussão}

A formação docente é um aspecto fundamental para a mudança de paradigmas no ensino na saúde, visto que os professores precisam saber mais que sua especialidade profissional e suas áreas de atuação. Para tanto, é necessário compreender a visão global da função docente, sobretudo no momento da preceptoria clínica, processo imprescindível na formação do futuro profissional $^{(14)}$. 
Apesar de todo o corpo de preceptores e/ou supervisores possuírem pós-graduação e um número considerável de mestres ou doutores, foi observado que alguns possuem como titulação máxima a residência médica. O fato que chama atenção é que os mesmos 03 pertencem à três diferentes grandes áreas da medicina (Pediatria, Clínica médica e Ginecologia e obstetrícia) o que sugere certa homogeneidade da amostra coletada.

O desenvolvimento docente, mesmo sendo referido pela maioria, observa-se uma vinculação direta com as disciplinas de pós-graduação latu e stricto sensu, o que sugere uma carga horária restrita. A literatura mostra que o professor da área da saúde geralmente não possui preparo específico no campo pedagógico - uns exercem a docência com base na formação didática que receberam em cursos de licenciatura, outros trazem sua experiência profissional para a sala de aula e outros não tem experiência didática nem profissional $^{(14-15)}$. Além disso, como aponta Mosorini ${ }^{(15)}$, ele sofre diversas pressões para a qualificação de seu desempenho, sobretudo didático:

[...] do governo com o fito de avaliar a qualidade do ensino superior, imposta pela instituição com o objetivo de obter credenciamento da mesma junto ao MEC e para captar os alunos e buscada pelo professor para a manutenção de seu emprego e aumento de remuneração, entre outros requisitos ${ }^{(15)}$.

Quanto ao tempo na função de preceptor e/ou supervisor, a metade dos pesquisados estão, segundo a classificação de Huberman apud Isaia $^{(12)}$, na fase de consolidação da competência pedagógica (fase inicial). A outra metade encontra-se na fase de diversificação/questionamento, o que sugere que boa parte dos profissionais tem como tônica a motivação e o dinamismo, necessidade de contribuir para a reformulação do sistema ${ }^{(12)}$. Estes resultados se assemelham aos encontrados por outros autores Lima e Vilela $^{(16)}$, e sugerem que o investimento nos docentes durante estas fases pode ser decisivo para o grupo se aproximar mais e melhor da docência. Huberman $^{(12)}$ ressalta que a fase de diversificação/questionamento (7 a 25 anos de docência) é a fase mais longa do professor e onde se encontram três tipos básicos: a) aqueles que investem seu potencial no desenvolvimento como docente, buscando diversificar seus métodos e práticas e as formas mais adequadas de aplicá-las no ensino; b) outros que se envolvem mais com o sistema administrativo, visando promover-se profissionalmente; c) aqueles que aos poucos reduzem seus compromissos com a docência, podendo 
abandoná-la ou exercer outra profissão paralela.

Foi observada satisfação com a função exercida como supervisor, entre os entrevistados. Marqueze ${ }^{(17)}$, em seu estudo, identificou dados relativos a fatores relacionais e profissionais, que apontavam a satisfação docente com o trabalho exercido, sendo, no entanto, necessário o desenvolvimento de trabalhos mais profundos voltados à temática da docência na preceptoria de Medicina.

A Educação Permanente é uma das competências estabelecidas pelas Diretrizes Curriculares Nacionais para os profissionais de saúde. Esta competência se apoia no conceito de ensino problematizador e de aprendizagem significativa, ou seja, ensino-aprendizagem embasado na produção de conhecimentos que respondam a perguntas que pertençam ao universo de experiências e vivências de quem aprende e que gerem novas perguntas sobre o ser e o atuar no mundo ${ }^{(1,18,19)}$.

No internato de medicina desta escola, dentre as ações/atividades que são realizadas durante o momento da preceptoria/supervisão, os profissionais foram unânimes com relação à indicação de leituras complementares, explicações orais e demonstrações de técnicas e procedimentos. Esses dados comprovam a importância da figura do preceptor/supervisor na função de articular teoria e prática, bem como aproximar o estudante do raciocínio clínico necessário à profissão, sobretudo no internato ${ }^{(13-14)}$.

Nos casos, tipo ou modo de planejamento da atividade encontrados nessa pesquisa, pode-se observar que o trabalho em equipe que engloba também os profissionais médicos de outras especialidades e os profissionais de outras especialidades que não médicas é praticado de forma muito restrita. Essa informação reflete a ausência de práticas interdisciplinares e interprofissional no cotidiano do internato de Medicina, o que se opõe ao que está proposto nas DCN (2014), bem como nos estudos acerca de Interdisciplinaridade na saúde, os quais apontam para a necessidade de atenção integral à saúde, o que só é possível diante do olhar interdisciplinar ${ }^{(7,8,10,20)}$.

O trabalho em equipe, executado em sua plenitude, perpassa a atuação interprofissional e o planejamento conjunto das ações, consolidando a EPS, competência que permite a reflexão sobre a prática e a solução de problemas cotidianos dos serviços de saúde, favorecendo a prestação de 
serviços conforme as reais demandas individuais e comunitárias ${ }^{(1,4,10,18)}$.

A educação interprofissional (EIP), onde estudantes de duas ou mais profissões aprendem sobre os outros, com os outros e entre si para possibilitar a efetiva colaboração e melhorar os resultados na saúde ${ }^{(10)}$, pode ser alcançada ao se priorizar o trabalho da competência de EPS. Esta estratégia permite o exercício do aprender a aprender e visa à reflexão sobre a prática. Esse conceito, oriundo da educação, ganhou status de Política na área de saúde no Brasil, com o objetivo de favorecer espaços conjuntos de reflexão sobre a prática, envolvendo diversos atores como docentes e discentes de diversas áreas, profissionais do serviço, gestão e usuários, com vistas a solucionar coletivamente os problemas emergidos da prática cotidiana. Para tanto, é necessário que ocorra de forma periódica, favorecendo, assim, a efetivação da integração ensino-serviço, viabilizando a aprendizagem significativa por parte dos futuros profissionais, despertando os trabalhadores para a reflexão crítica do serviço e oferecendo à comunidade uma atenção integral $^{(1-7,18)}$.

\section{Conclusão}

O desenvolvimento docente dos preceptores e/ou supervisores encontrase diretamente vinculado a disciplinas de pós-graduação latu e stricto sensu, o que sugere uma carga horária restrita específica para a docência, durante sua formação.

No que tange à preceptoria e/ou supervisão propriamente dita, os profissionais dedicam de 13 a 24 horas semanais à mesma em metade dos entrevistados, realizam atividades que englobam, dentre outras, a indicação de leituras complementares, a explicação oral e a demonstração de técnicas e procedimentos junto aos internos. Essas atividades envolvem, predominantemente, os trabalhos isolados entre preceptor/supervisor e aluno e entre o preceptor/supervisor, aluno e paciente/usuário sendo em sua totalidade fruto de um planejamento conjunto entre o preceptor/supervisor e o aluno. Não há presença de profissionais e/ou estudantes de outras áreas, além da medicina.

A análise dos constructos revelam que é necessário investimento em desenvolvimento docente visando uma prática pedagógica mais problematizadora e significativa, como metodologia de ensino-aprendizagem. Espaços esses que possibilitem a EIP, 
capazes de provocar nestes docentes e, consequentemente, nos estudantes, novas reflexões, novos caminhos, novos sentidos para a prática com o exercício da EPS.

Por fim, o estudo aponta a necessidade de ampliar os conhecimentos sobre o tema e suas diversas concepções, em especial o aprofundamento das discussões teóricoconceituais e empíricas em torno da EPS, da EIP e suas repercussões nas práticas de saúde. Também identifica a necessidades de conhecer as iniciativas de interdisciplinaridade e interprofissionalidade, reconhecendo que são complementares.

\section{Referências}

1. Ceccim RB, Feuerwerker LCM. O quadrilátero da formação para a área de saúde: ensino, gestão, atenção e controle social. Physis (Rio de Janeiro). 2004; 14 (1): 41-65.

2. Beinner MA, Beinner RPC. The profile of professionals in health and education fields at work in their communities. Ciênc. Saúde Colet. 2004;9 (1): 77-83.

3. Rossoni E, Lamperti J. Formação de profissionais para o Sistema Único de Saúde e as diretrizes curriculares. Bol. Saúde. 2004; 18 (1): 87-98.

4. Feuerwerker L. Educação dos profissionais de saúde hoje: problemas, desafios, perspectivas, e as propostas do
Ministério da Saúde. Rev. ABENO. 2003; 3 (1): 24-7.

5. Haddad AE, Pierantoni CR, Ristoff D, Xavier IM, Giolo J, Silva LB. A Trajetória dos Cursos de Graduação na Saúde: 1991 a 2004. Brasília: Instituto Nacional de Estudos e Pesquisas Educacionais Anísio Teixeira; 2006. Disponível em: http://www.publicacoes. inep.gov.br/arquivos/\%7B64512EC6-B B2E-43FE-B4BD-0F1AA7769428\%7D _Texto_de_Referencia.pdf. Acesso em: 16 de maio 2016.

6. Faria RMB. Institucionalização da política de educação permanente para o Sistema Único de Saúde: Brasil, 19972006. [Dissertação]. Universidade de São Paulo, São Paulo; 2008.

7. Ministério da Educação (BR). Conselho Nacional de Educação. Câmara de Educação Superior. Resolução CNE/CES 3/2014. Institui Diretrizes Curriculares Nacionais do Curso de Graduação em Medicina e dá outras providências. Diário Oficial da União, Brasília: Ministério da Educação; 2014.

8. Universidade Federal de Alagoas. Faculdade de Medicina. Projeto Pedagógico do Curso de Medicina. Maceió - Alagoas; 2013 [citado 2016 mar. 15]. Disponível em: http://www.famed.ufal.edu.br

9. Missaka HA. Prática pedagógica dos preceptores do internato em emergência e medicina intensiva de um serviço público não universitário. [Dissertação]. Universidade Federal do Rio de Janeiro, Rio de Janeiro, 2010 [citado 2016 mar. 10]. Disponível em: http://www.nutes. ufrj.br/mestrado/arquivos/dis.HMissaka. pdf

10. World Health Organization. Framework for action on interprofessional education \& 
collaborative practice. Report. Geneva; 2010 [citado 2016 abr. 21]. Disponível em: http://apps.who.int/iris/bitstream/10 665/70185/1/WHO_HRH_HPN_10.3_e ng.pdf?ua=1.

11. Botti, SHO, Rego, S. Preceptor, Supervisor, Tutor e Mentor: Quais são os seus papéis. Ver. Bras. Educ. Méd. 2008; 32 (3): 363-73.

12. Isaia, SMA. Professor universitário no contexto de suas trajetórias como pessoa e profissional. In: Morosini MC. Professor do ensino superior: identidade, docência e formação. Brasília, INEP; 2000 [citado 2016 jan. 15]. 21-33. Disponível em: http://www.publicacoes.inep.gov.br/port al/download/197

13. Trajman AA. Preceptoria na rede básica da Secretaria Municipal de Saúde do Rio de Janeiro: opinião dos profissionais de saúde. Bras. Educ. Méd. 2009;33 (1): 24-32.

14. Costa NMSC. Docência no ensino médico: por que é tão difícil mudar? Bras. Educ. Méd.2007; 31 (1):21-30.

15. Morosini MC. Docência universitária e os desafios da realidade nacional. In: Morosini MC. Professor do ensino superior: identidade, docência e formação. Brasília, INEP; 2000 [citado 2016 mar. 15]. p. 21-33. Disponível em: <http://www.publicaco es.inep.gov.br/portal/download/197>.

16. Lima BPS, Vilela RBV. Características e desafios docentes na supervisão de estágio em fonoaudiologia. Rev. CEFAC. 2014; 16(6): 1962-71.

17. Marqueze EC, Moreno C. R. C. Satisfação no trabalho e capacidade para o trabalho entre docentes universitários. Psicol. Estud. 2009; 14 (1): 75-82.
18. Ceccim RB. Educação Permanente em Saúde: desafio ambicioso e necessário. Interface (Botucatu). 2005; 9 (16): 161-77.

19. Ceccim RB. Educação Permanente em Saúde: descentralização e disseminação da capacidade pedagógica na saúde. Ciênc. Saúde Colet. 2005; 10 (4): 975-86.

20. Japiassu H. Interdisciplinaridade e patologia do saber. Rio de Janeiro: Imago; 1976. 